\title{
XMRV—contaminant, not cause?
}

D oubts about the association between xenotropic murine leukemia virus-related virus $(\mathrm{XMRV})$ and prostatic malignancy have been lent great weight by several recently published papers. The new evidence indicates that XMRV is an artifact generated during production of prostate cancer cell lines.

The hypothesis that a virus might induce neoplastic transformation of prostate cells is a sound one. Viruses are known to cause cancer of the cervix and liver, and the antiviral gene RNASEL has been linked to hereditary prostate cancer. However, XMRV has been detected at widely disparate frequencies in prostate cancer populations-from $0 \%$ to $27 \%$.

A US-based team sought to shed light on this discrepancy by investigating how XMRV first arose. The group focused their efforts on the human prostate cancer cell line CWR22Rv1, a widely used model of androgen-independent disease that produces infectious XMRV. In order to establish this line in the early 1990s, cells had been harvested from a primary prostate tumor and used to generate a xenograft (CWR22). Serial xenograft transplants in immunodeficient mice were then performed before cells were harvested for propagation in vitro.

Vinay Pathak and colleagues began their investigation by estimating the relative quantities of XMRV in the cell line and its xenograft precursor. At 2,000 copies per 100 cells and $0-3$ copies per 100 cells, respectively, XMRV levels seemed to have increased dramatically during serial passage.

"For a retrovirus to cause cancer, almost every cancer cell should harbor the virus", team member Hsing-Jien Kung said in a press statement. "When we found the virus was not in the early passages of the tumor cells, but plentiful in the later ones, it made us suspicious that XMRV was not the cause of the original tumor".

Strikingly, xenografts passaged at the very earliest stages, which did not contain XMRV, did harbor a previously undescribed replication-deficient provirus dubbed 'PreXMRV-1' by Pathak, Kung and co-workers. A search for other XMRV-related proviruses revealed the presence of PreXMRV-2. Experimental evidence supports a murine origin for both proviruses.

"When we put [the gene sequences of the two proviruses] next to each other, they fit together like a puzzle. We knew immediately that they had generated XMRV," Pathak told the press.

Does this 'eureka moment' herald the end of the line for the XMRV-prostate cancer express? Pathak certainly thinks so. He told Medscape Medical News that "XMRV has nothing to do with human prostate cancer; the association is an artifact of laboratory contamination".

This opinion is seconded by the authors of another paper, published in Oncotarget. The team, led by Eugene Kandel from the Roswell Park Cancer Institute in New York State, used a robust and specific PCR strategy to look for XMRV. Their results mimicked those of Pathak et al.-XMRV was present in CWR22Rv1 cells, but not in the earlypassage xenograft of origin. "We had to conclude that the virus was not present in the original patient", says Kandel.

These findings have far-reaching implications, beyond XMRV and prostate cancer causality. Kandel points out that the presence of XMRV in CWR22Rv1 cells probably has a fundamental impact on their properties, thereby casting doubt on the relevance of findings obtained using this very popular preclinical model. "I wouldn't be surprised if some conclusions about the properties of prostate cancer cells would have to be revisited and reevaluated based on this knowledge."

Prostate cancer is not the only disease to have received the XMRV-debunking treatment. Pathak and colleagues' Science paper was accompanied by another study,

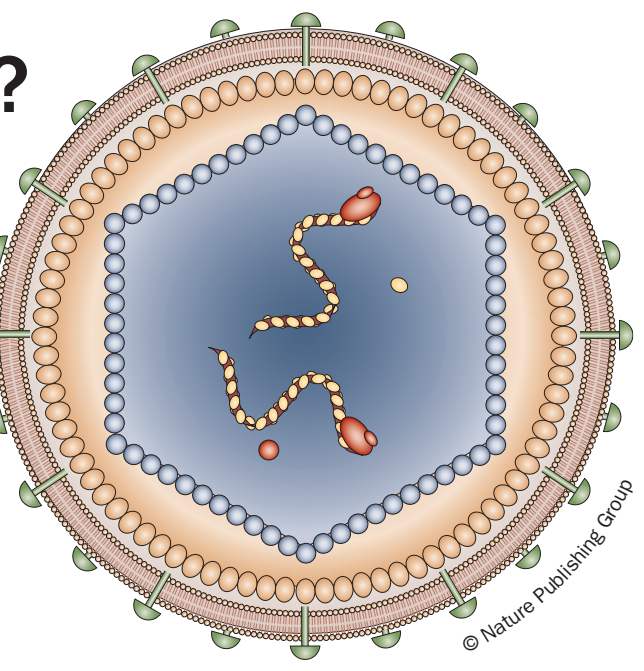

a re-examination of XMRV in people with chronic fatigue syndrome (CFS).

Konstance Knox and co-workers failed to detect XMRV in the blood of any of 61 CFS patients, 43 of whom had been previously categorized as XMRV-positive in the seminal work of Vincent Lombardi et al. Based in part on the conclusion of Knox et al. that the link between XMRV and CFS is a spurious one arising from laboratory contamination, the Editors of Science have requested retraction of the original Lombardi paper. The authors have refused this request. They assert that important differences in experimental techniques used by the two groups are at the root of the discrepant outcomes.

Hopefully, ongoing studies sponsored by the National Institutes of Health will bring this saga to a swift and definitive close. If the XMRV-based hopes of patients are dashed as a result, let's hope that rapid refocusing of research efforts will reveal a new standard to which we can more fruitfully pledge allegiance.

Suzanne J. Farley 